\title{
The Lateral Habenula Has Vesicles That Accumulate Either GABA or Glutamate
}

Shiliang Zhang ${ }^{1}$, David H Root ${ }^{2}$, David J. Barker ${ }^{3}$ and Marisela Morales ${ }^{4}$

1. Electron Microscopy Core, National Institute on Drug Abuse, Baltimore, United States.

2. Neuronal Networks Section, Integrative Neuroscience Research Branch, National Institute on Drug Abuse, Baltimore, United States.

3. Neuronal Networks Section, Integrative Neuroscience Research Branch, National Institute on Drug Abuse, Baltimore, United States.

4. Neuronal Networks Section, Integrative Neuroscience Research Branch, National Institute on Drug Abuse, Baltimore, United States.

The lateral habenula $(\mathrm{LHb})$ is involved in reward, aversion, addiction and depression through descending interactions with several structures, including the ventral tegmental area (VTA). Our recent studies have revealed that within the LHb, single axon terminals from VTA neurons co-transmit glutamate and GABA via a previously unrecognized combinatorial synaptic architecture. In this combinatorial synaptic architecture, single axon terminals co-express vesicular glutamate transporter 2 (VGluT2) and vesicular GABA transporter (VGaT), establish both asymmetric and symmetric synapses, express AMPA receptors postsynaptic to asymmetric synapses, and express GABA-A receptors postsynaptic to symmetric synapses [1]. The spatial segregation of asymmetric and symmetric synapses within single mesohabenular axon terminals suggests that glutamate and GABA are released at different synapses and from distinct synaptic vesicles. However, it remains to be tested if dual glutamatergicGABAergic neurons store glutamate (via VGluT2) and GABA (via VGaT) in the same vesicular populations. To determine whether glutamate and GABA are stored and released from the same or from a different pool of vesicles, we purified synaptic vesicles from the LHb of Sprague-Dawley rats $(n=80)$. By dual immunolabeling of these isolated synaptic vesicles and their analysis under electron microscopy, we detected two distinct pools of vesicles: a pool of vesicles containing VGluT2immunolabeling and another pool containing VGaT-immunolabeling. We found that from a total of 2,815 immunolabeled vesicles $58.92 \pm 0.97 \%$ expressed VGluT2, $38.92 \pm 0.81 \%$ expressed VGaT, and a very small proportion $(2.15 \pm 0.29 \%)$ appeared to co-label for VGluT2 and VGaT. The lack of coexistence of VGluT2 and VGaT at the vesicular level was next confirmed by the lack of coimmunoprecipitation of VGluT2 and VGaT from the isolated vesicles. Consistent with our ultrastructural findings, protein preparations of vesicles immunoprecipitated with antibodies against VGluT2 showed vesicular immunodetection of both VGluT2 and the vesicular marker synaptophysin, but not VGaT. Therefore, we conclude that within the LHb, accumulation of glutamate by VGluT2 occurs in vesicles different from those that accumulate GABA by VGaT, and glutamate and GABA are released from separate synapses within single axon terminals.

Key words: VGluT2, VGaT, glutamate, GABA, lateral habenula, VTA, co-transmission [2].

References:

[1] DH Root et al, Nature Neuroscience 17 (2014), p. 1543.

[2] The authors acknowledge funding from the NIDA-IRP. 

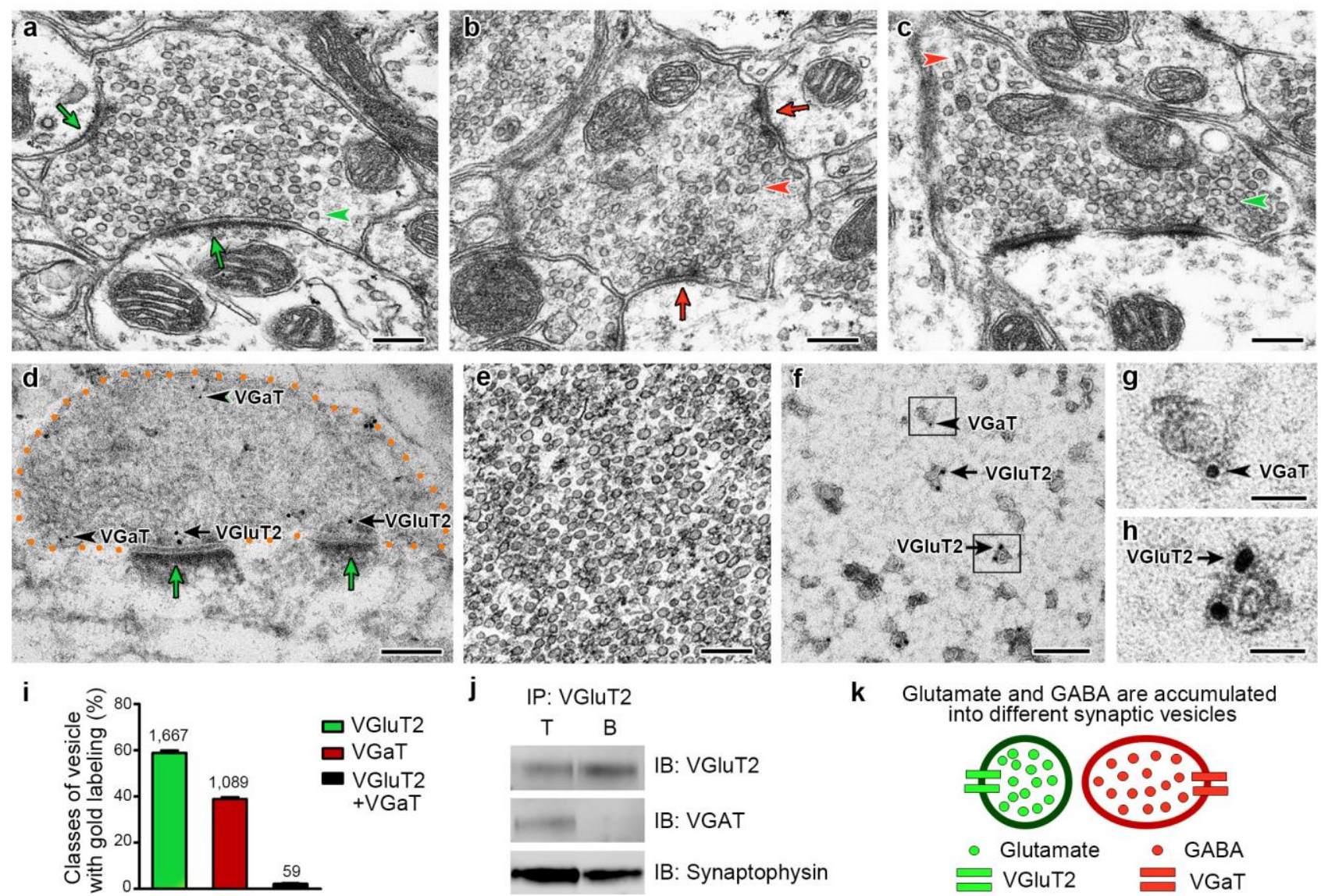

j IP: VGIUT2

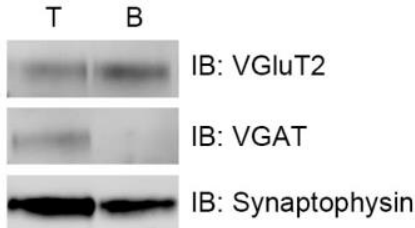

k Glutamate and GABA are accumulated into different synaptic vesicles

Figure 1. Glutamate and GABA are stored and released from distinct subpopulations of synaptic vesicles.

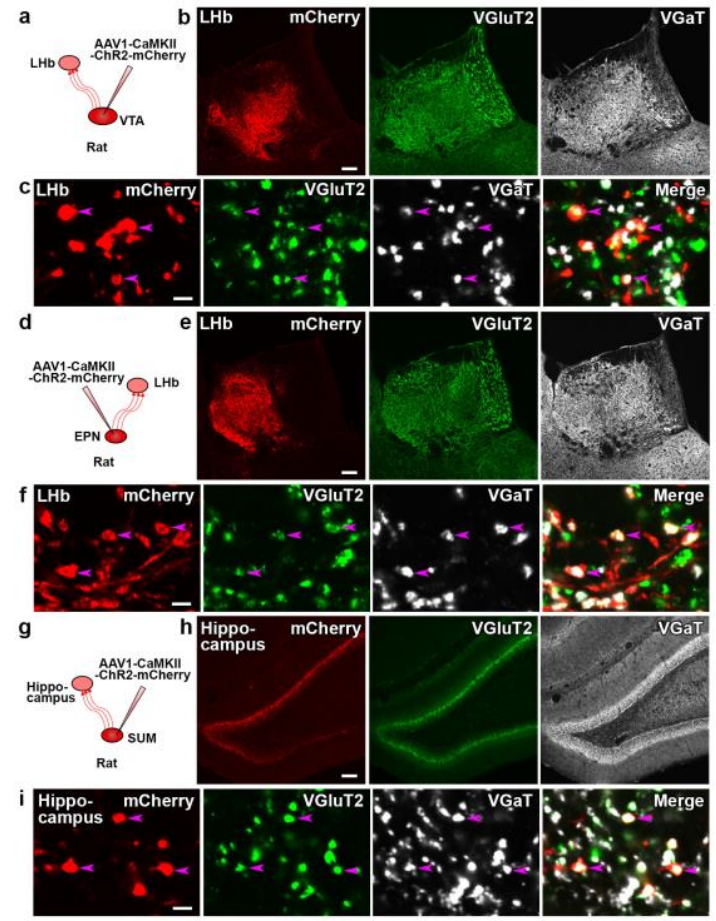

Figure 2. Most LHb or hippocampus axon terminals derived from VTA, EPN or SUM co-express VGluT2 and VGaT. 